\title{
Empirische Evidenz zu den Wirkungen der Einführung des G-DRG-Systems
}

Ricarda Milstein und Jonas Schreyögg

2.1 Einleitung - 26

2.2 Eine kurze Chronologie der Fallpauschaleneinführung - 27

2.3 Studien zu Wirkungen der Einführung des G-DRG-Systems und anderer Determinanten auf die Fallzahlentwicklung - 30

2.4 Untersuchung der Auswirkungen des Fallpauschalensystems auf die technische und Kosteneffizienz - 33

2.5 Auswirkungen des Fallpauschalensystems auf die Behandlungsqualität - 34

2.6 Diskussion: Die Auswirkungen der DRG-Einführung bleiben bei zahlreichen Indikatoren unklar - 35

Literatur - 36 


\section{- Zusammenfassung}

Das deutsche Fallpauschalensystem wurde 2000 beschlossen und ist seit 2004 das verpflichtende Vergütungssystem für alle Akutkrankenhäuser. Seit dem Ende der Budgetneutralität 2005 setzt es Anreize an die Leistungserbringung. Es sollte die Wirtschaftlichkeit, Transparenz und Effizienz des deutschen Krankenhaussystems verbessern und gleichzeitig zu einer Reduktion der Verweildauer und einem Abbau der Bettenkapazitäten führen. In den Folgejahren sank die durchschnittliche Verweildauer, aber weniger stark als zuvor. Zeitgleich verzeichnete Deutschland eine Zunahme der Fallzahlen und der Krankenhausausgaben. Derzeit sind die Auswirkungen der Fallpauschaleneinführung auf die Leistungserbringung wenig bekannt. Forschungsergebnisse konnten zeigen, dass Veränderungen auf der Nachfrageseite eine geringe Rolle spielten. Stattdessen kommt den Veränderungen in der Angebotsstruktur als Reaktion auf Preisänderungen eine größere Rolle zu, wenngleich dieser Effekt je nach Diagnosegruppe unterschiedlich ist. Studien fanden zudem Hinweise auf Upcoding von Patienten. Der Effekt des Fallpauschalensystems auf die Krankenhauseffizienz und Qualität der Leistungserbringung ist hingegen kaum bekannt. Das Fehlen einer Kontrollgruppe erschwert die Ermittlung kausaler Effekte. Zudem wurden die meisten Studien auf Basis von aggregierten Daten erstell und konnten nicht mit Daten außerhalb des stationären Sektors verknüpft werden. Dies schmälert ihre Aussagekraft. Das Fehlen belastbarer Untersuchungen erschwert Empfehlungen zielgerichteter, notwendiger Reformen des Fallpauschalensystems.

The German DRG system was introduced in 2000 and has been the obligatory reimbursement system for all acute care hospitals since 2004. It was intended to improve the transparency and efficiency of Germany's hospitals, reduce the average length of stay and the number of beds. In the years following its introduction, the average length of stay decreased, but less so than before. At the same time, Germany recorded an increase in the number of cases and in hospital expenditures. To date, the effect of the DRG introduction on the provision of services in Germany's hospital sector remains largely unknown. Research findings showed that changes on the demand side yield little explanatory power. Instead, changes in the hospital supply structure following price changes play a greater role, albeit to a varying degree. Studies also found some evidence of upcoding. The effect of the DRG system on hospital efficiency and quality of care has not been properly investigated yet. Evaluations suffer from the lack of a control group hampering the investigation of causal effects. Furthermore, most evaluations used aggregate data which could not be merged with non-inpatient data. The lack of reliable studies makes it difficult to recommend targeted, necessary reforms of the DRG introduction.

\subsection{Einleitung}

Mit der 2000 beschlossenen, 2003 begonnenen und 2004 bundesweit verpflichtend eingeführten Umstellung der Krankenhausvergütung auf ein Fallpauschalensystem folgte Deutschland einem internationalen Trend. Es vollendete damit den Weg, die Vergütung nicht mehr an die Verweildauer, sondern nunmehr an die Fallzahl zu knüpfen, den es 1993 durch die Einführung der Budgetierung beschritten und 1995 durch die Einführung pauschalisierter, leistungsbezogener Entgelte ausgebaut hatte. Das neue Fallpauschalensystem, beziehungsweise German Diagnosis-RelatedGroups-System (G-DRG-System), sollte nach der Maxime des "gleichen Preises für gleiche Leistung" die Transparenz, Effizienz und Wirtschaftlichkeit der deutschen Krankenhäuser steigern, die Bettenzahl reduzieren und eine Reduzierung der Verweildauern erwirken (Deutscher Bundestag 1999, 2001). Das vorausgegangene Mischsystem aus Fallpauschalen, Sonderentgelten und Pflegesätzen galt hierfür als gescheitert (Deutscher Bundestag 2001).

Diese Vergütungsart setzt klare Anreize an die Leistungserbringung von Krankenhäusern 
(Schreyögg 2019; Ellis und McGuire 1996). Erstens wird die Fallzahl zum wichtigsten Parameter der Vergütung. Dadurch, dass Krankenhäuser nun auf Basis der Fallzahl und nicht mehr auf Per-Diem-Basis nach Tagessätzen vergütet werden, setzen sich mehr Fälle in höhere Erlöse um. Zweitens darf angenommen werden, dass ein Fallpauschalensystem zu einer Effizienzsteigerung führt. Da die Preise, beziehungsweise Relativgewichte, pro DRG unter den Krankenhäusern, die ihre Kostendaten zur Preisberechnung zur Verfügung stellen, gemittelt werden, treten Krankenhäuser in einen Effizienzwettbewerb untereinander (Yardstick-Wettbewerb) (Shleifer 1985). Krankenhäuser, deren Kosten für eine Fallpauschale oberhalb der Vergütung liegen, werden angereizt, ihre Kosten zu senken - beispielsweise durch die Vermeidung von unnötigen Untersuchungen, Reduzierung der Verweildauer, des Personaleinsatzes und durch technische Innovationen. Krankenhäuser mit Ausgaben unterhalb der Vergütung werden für ihre effiziente Leistungserbringung belohnt. Drittens setzt das DRG-System einen Anreiz zur Reduktion der Verweildauer, da sich kürzere Verweildauern in geringe Kosten - wenn möglich unterhalb der DRG-Vergütung - übersetzen und somit höhere Erlöse für das Krankenhaus ermöglichen. Zudem müssen sie Patienten entlassen, um neue Patienten aufnehmen und somit neue Fallpauschalen abrechnen zu können. Darüber hinaus sind die Kurzliegerabschläge beziehungsweise Langliegerzuschläge so ausgestaltet, dass eine Abweichung von der vorgesehenen Verweildauer finanziell in vielen Fällen unattraktiv ist.

Die erste vorsichtige Zwischenbilanz der Vorreiter des Fallpauschalensystems, beispielsweise der Vereinigten Staaten, wirkte vielversprechend (Davis und Rhodes 1988; Coulam und Gaumer 1992). In den ersten Jahren nach Einführung des Fallpauschalensystems für Medicare im Jahr 1983 schien das DRG-System die Ausgaben- und Kostensteigerungen des vorhergehenden Fee-for-Service-Systems eingedämmt zu haben (Russell und Manning 1989; Feder et al. 1987). Erste Untersuchungen stellten eine Senkung der Verweildauer bei gleichbleibender Behandlungsqualität fest (Sager et al. 1989), wenngleich sich der Verdacht auf unerwünschte Nebeneffekte abzeichnete und die Evaluationsperiode sehr kurz war (Coulam und Gaumer 1992).

Ziel des vorliegenden Kapitels ist es, einen Überblick zu den bisherigen empirischen Evaluationen der Wirkungen des G-DRG-Systems zu geben. Dabei wird auch auf die ursprünglichen Ziele des G-DRG-Systems eingegangen. Nach einem kurzen historischen Abriss der Entwicklung des G-DRG-Systems und dessen ursprünglich formulierten Zielen folgt ein kurzer Überblick über die Entwicklung der Kernindikatoren. Im zweiten Teil des Kapitels geben wir einen Überblick über die empirischen Ergebnisse bisheriger Evaluationsstudien des GDRG-Systems. Anschließend werden diese Ergebnisse eingeordnet und diskutiert.

\subsection{Eine kurze Chronologie der Fallpauschaleneinführung}

Aufbauend auf der theoretischen Struktur und den positiven ersten Erfahrungen anderer Industrienationen mit Fallpauschalensystemen sollte das als ineffizient geltende deutsche Krankenhaussystem grundlegend überholt werden. Der Start in Deutschland war allerdings ein wenig holprig und von einer Reihe von Ersatzvornahmen geprägt. Den Ausgangspunkt nahm die DRG-Einführung mit dem Gesetz zur Reform der gesetzlichen Krankenversicherung vom 22. Dezember 1999 (GKVGesundheitsreform 2000, Deutscher Bundestag 1999), mit dem der Deutsche Bundestag die Einführung eines „durchgängigen, leistungsorientierten und pauschalisierten Vergütungssystems mit vereinbarten Mengen“ für vollund teilstationäre Leistungen ab dem 1. Januar 2003 beschied (Deutscher Bundestag 1999). Die zudem im Gesetzesentwurf vorgesehene Umstellung von einer dualistischen auf eine monistische Krankenhausfinanzierung (Deutscher Bundestag 1999) scheiterte hingegen an der Zustimmung des Bundesrates. 
Bis Mitte 2000 sollten sich die damaligen Spitzenverbände der Krankenkassen und der Verband der Privaten Krankenversicherung mit der Deutschen Krankenhausgesellschaft auf einen Grundrahmen des Fallpauschalensystems verständigen und bis Ende $2001 \mathrm{Be}$ wertungsrelationen sowie $\mathrm{Zu}$ - und Abschläge festlegen. Zum 27. Juni 2000 einigte sich die Selbstverwaltung auf eine Übernahme des Australian Refined DRG-Systems (AR-DRGSystem), das als sehr transparent, medizinisch weiter entwickelt, leistungsgerechter und weniger manipulationsanfällig als alternative Systeme galt (Leber et al. 2001; Roeder et al. 2008). Die Verhandlungen der weiteren Ausgestaltung endeten jedoch mit der Aufkündigung der Verhandlungen von Seiten der Deutschen Krankengesellschaft am 24. Juni 2002 (Deutscher Bundestag 2002). Das Bundesministerium intervenierte infolgedessen mit dem 2002 verabschiedeten Fallpauschalengesetz (FPG) (Deutscher Bundestag 2001), gab der Selbstverwaltung eine Karenzzeit bis zum 01.01.2004 zur Einführung und legte den Fallpauschalenkatalog mittels Fallpauschalenverordnung (Verordnung zum Fallpauschalensystem für Krankenhäuser (KFPV)) vor. Damit konnten Krankenhäuser auf freiwilliger Basis auf das budgetneutrale „Optionsmodell 2003“ umstellen, dem gut 1.000 Krankenhäuser folgten (Deutscher Bundestag 2004). Nach einem erneuten Scheitern der Verhandlungen und deren Aufkündigung durch die DKG vom 2. Juli 2003 wiederholte das Ministerium dieses Procedere für das folgende Jahr mit der erneuten Vorlage der Fallpauschalenverordnung (KFPV 2004). Damit folgten die restlichen Krankenhäuser mit der Umstellung der Vergütung auf das weiterhin budgetneutral ausgestaltete Fallpauschalensystem (Deutscher Bundestag 2004).

2004 einigten sich die Partner der Selbstverwaltung erstmals auf eine Fallpauschalenvereinbarung (AOK-Bundesverband et al. 2004). Gleichzeitig läutete dieses Jahr das Ende der Budgetneutralität und den Beginn der Konvergenzphase ein. Verhandlungen um Landesbasisfallwerte scheiterten jedoch und wurden per Verordnung vom Bundesministeri- um für Gesundheit und Soziale Sicherung vorgegeben (KFPV 2005). Dem folgte mit dem 2004 verabschiedeten Zweiten Fallpauschalenänderungsgesetz (2. FPÄndG) (Deutscher Bundestag 2004) eine sukzessive Verlängerung der Konvergenzphase von 2007 auf schlussendlich 2010. Das Krankenhausfinanzierungsreformgesetz (KHRG) sah eine Anpassung der Landesbasisfallwerte der Ende 2009 noch sehr heterogenen Landesbasisfallwerte an einen einheitlichen Basisfallwertkorridor vor (Deutscher Bundestag 2008). Das 2016 in Kraft getretene Krankenhausstrukturgesetz (KHSG) sieht eine weitere Annäherung an einen Bundesbasisfallwert mit einer Verengung des Korridors bis zum Jahr 2021 vor (Deutscher Bundestag 2015).

\section{- Moderate Reduzierung der Verweildauer bei gleichzeitigem Fallzahlanstieg}

Das Ziel, die Verweildauer zu reduzieren, scheint auf den ersten Blick überwiegend eingetreten zu sein (•Abb. 2.1): Seit Einführung des Fallpauschalensystems sank die Verweildauer um fast $15 \%$ von 8,7 Tagen zum Zeitpunkt der verpflichtenden Einführung und dem Beginn der Konvergenzphase 2005 auf 7,3 Tage im Jahr 2017 (Statistisches Bundesamt 2018). Hiermit reiht sich Deutschland in die Erfahrungen anderer Länder wie beispielsweise England, Österreich und den Vereinigten Staaten ein, die einen ähnlichen Effekt verzeichnen konnten (Theurl und Winner 2007; Kahn et al. 1990; Farrar et al. 2009). Allerdings ist zu konstatieren, dass der Rückgang der Verweildauer vor der Einführung des Fallpauschalensystems deutlich prononcierter war als nach der Einführung: Zwischen 1991 und 2005 sank die Verweildauer um fast $40 \%$, von durchschnittlich 14,0 Tagen auf 8,7 Tage. Dies deckt sich mit Erfahrungen in beispielsweise der Schweiz. Dort war der Rückgang nach Einführung der Fallpauschalen nicht stärker als vorher (Kutz et al. 2019). Es ist demnach unklar, ob die Einführung des G-DRG-Systems den Rückgang der Verweildauer befördert hat.

Zeitgleich markiert die Einführung des Fallpauschalensystems eine Rückkehr zum An- 


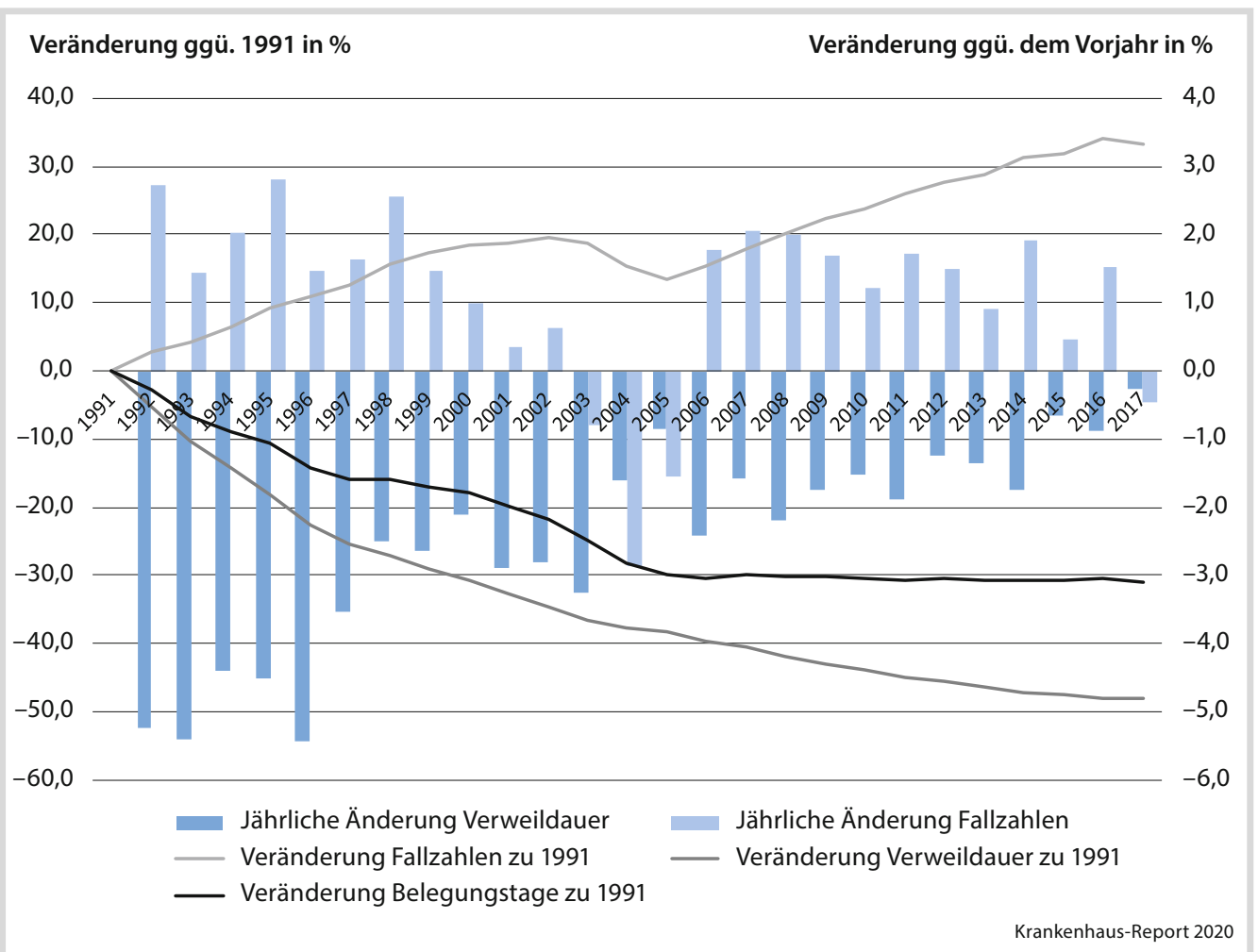

- Abb. 2.1 Veränderung von Kernindikatoren in \% zum Vorjahr/zu 1991 (Quelle: Statistisches Bundesamt 2018)

stieg der stationären Fallzahlen (siehe die Säulen in - Abb. 2.1). Zwischen 1991 und 2002 stieg die Fallzahl um $20 \%$ mit einer durchschnittlichen jährlichen Steigerung von 1,6\%. Verlagerungen von Operationen in den ambulanten Sektor führten zwischen 2002 und 2005 kurzzeitig zu einer Fallzahlreduktion auf das Niveau von 1997 mit einem Rückgang von $5 \%$ gegenüber 2002. Mit der verpflichtenden Einführung der Fallpauschalen und dem Einleiten der Konvergenzphase kehrte der jährliche Fallzahlanstieg zu seiner vorherigen Dynamik zurück. Über den Zeitraum von 2005 bis 2017 stieg die Fallzahl wieder um insgesamt rund $18 \%$ an, mit einer durchschnittlichen jährlichen Wachstumsrate von 1,4\%. Der Anstieg ist jedoch je nach Verweildauer heterogen. Zwischen 2007 und 2016 nahm die Zahl der Fälle mit Verweildauern zwischen einem und drei Tagen um rund $43 \% \mathrm{zu}$, gefolgt von
„Stundenfällen“ mit einem Anstieg von $18 \%$. Die Zahl der Fälle mit Verweildauern zwischen vier und sechs Tagen nahm moderat um rund $9 \%$ zu. Demgegenüber sank die Zahl der Fälle mit Verweildauern von über sechs Tagen um $8 \%$ (Sachverständigenrat zur Begutachtung der Entwicklung des Gesundheitswesens 2018).

Die Fallzahlsteigerung der letzten Jahre hat zu einer grundlegenden Kritik am deutschen Fallpauschalensystem geführt. Strittig ist, inwieweit die Fallzahlsteigerung kausal auf das Fallpauschalensystem und seine Ausgestaltung attribuiert werden kann. Einerseits kann der Zuwachs der Fälle auf eine gestiegene Nachfrage zurückzuführen sein, beispielsweise durch eine zunehmende Alterung der Gesellschaft und den damit einhergehenden Zuwachs an (Multi-)Morbidität. Zudem können sich darin der medizinische Fortschritt und mithin die Erweiterung des medizinischen Leistungsspek- 
trums niederschlagen. Gleichzeitig ist es denkbar, dass Krankenhäuser die Menge in finanziell lukrativen Diagnosegruppen ausweiten und die Nachfrage somit aktiv induzieren (Coulam und Gaumer 1992; Dafny 2005). Ebenso könnten Krankenhäuser den Kodierspielraum ausnutzen und Patienten in finanziell attraktivere Fallpauschalen einordnen als medizinisch naheliegend. Zudem ist umstritten, inwieweit sich die Einführung des DRG-Systems in Effizienzgewinnen - beispielsweise einem konstanten Ressourceneinsatz bei steigender Fallzahl - niedergeschlagen hat. Ebenso strittig ist, ob das Fallpauschalensystem zu Veränderungen der Versorgungsqualität geführt hat, beispielsweise indem Patienten zu früh entlassen werden.

Im Folgenden werden wissenschaftliche Untersuchungen zum G-DRG-System zusammengefasst, die diesen in der internationalen Literatur zu DRG-Systemen formulierten Punkten nachgehen. Dabei wird die deutsche Erfahrung mit jener in ausgewählten Industrienationen kontrastiert.

\subsection{Studien zu Wirkungen der Einführung des G-DRG-Systems und anderer Determinanten auf die Fallzahlentwicklung}

Der Verdacht der ungerechtfertigten Mengenausweitung infolge der G-DRG-Einführung und die Diskussion möglicher Gegenmaßnahmen rückte Anfang dieses Jahrzehnts in den politischen Fokus. Dementsprechend haben sich verschiedene Untersuchungen der Frage gewidmet, ob die Steigerung der Fallzahlen auf eine gestiegene Nachfrage der Bevölkerung zurückzuführen sei, ob medizinisch-technische Innovationen ein größeres Leistungsangebot ermöglicht haben oder ob die Nachfrage durch die Leistungserbringer selbst als Reaktion auf Anreize des G-DRG-Systems induziert wurde (Augurzky et al. 2012; Kumar und Schoenstein 2013; Blum und Offermanns 2012).
Veränderungen in der Nachfragestruktur können durch verschiedene Entwicklungen verursacht werden. Diese umfassen grundsätzliche Bevölkerungszu- und abnahmen, eine zunehmende (Multi-)Morbidität der Bevölkerung, beispielsweise durch die Alterung der Gesellschaft und eine Änderung des Lebenswandels, sowie eine Zunahme der Anzahl von Patienten im letzten Lebensjahr. Letztere Gruppe wirkt besonders kostensteigernd für die $\mathrm{Ge}$ samtausgaben des Gesundheitssystems, weswegen ein hoher Beitrag dieser Gruppe zur Mengenentwicklung naheliegend wäre.

\section{- - Die Nachfrageseite hat einen begrenzten Einfluss auf die Fallzahlsteigerung}

Die DRG-Begleitforschung, die verpflichtend zur Einführung des Fallpauschalensystems in Auftrag gegeben wurde, ist zu diesem Zweck wenig aussagekräftig. Die Autoren zeigen, dass die Fallzahlentwicklung in etwa parallel zur Entwicklung der Altersstruktur verläuft. Da den Autoren lediglich aggregierte stationäre Daten zur Verfügung gestellt wurden, ist eine informative Aussage schwer möglich (Fürstenberg et al. 2013). Aussagen über den Einfluss von Morbiditätsveränderungen auf die Fallzahlsteigerung und eine feinere Untersuchung der Fallzahlsteigerung in einzelnen DRGs bzw. MDCs ist damit nicht möglich. Ein Gutachten des Deutschen Krankenhausinstituts (DKI) kommt zu dem Schluss, dass die Mengenentwicklung des Fallpauschalensystems stark von Veränderungen der Nachfrageseite getrieben wird (Blum und Offermanns 2012). Sie verwenden die Bevölkerungsstatistik sowie die aggregierten Diagnosedaten des Statistischen Bundesamtes von 2004 bis 2010 und simulieren für neun Altersgruppen und sechs virtuelle BasisDRGs, wie sich die Fallzahl bei einer Krankenhaushäufigkeit des Ausgangsjahres 2004, aber fortschreitender Bevölkerungszunahme entwickelt hätte, und umgekehrt. Für fünf von sechs virtuelle Basis-DRGs kommen die Autoren zu dem Schluss, dass die Bevölkerung der Haupttreiber der Fallzahlen ist, weil die simulierten Werte etwa deckungsgleich mit der tatsächlichen Fallsteigerung sind. Zudem führen 
sie die Zunahme der Fallschwere auf die sich kontinuierlich verbessernde Indikationsqualität und den technischen Fortschritt zurück. Letzteren stellen sie beispielhaft anhand der kathetergestützen Aortenklappenimplantation dar. Es bleibt jedoch unklar, inwieweit sich dies verallgemeinern lässt.

Dem widerspricht ein Gutachten von $\mathrm{Au}$ gurzky et al. (2012), das der Nachfrageseite einen untergeordneten Beitrag zur Mengenausweitung zuweist. Mittels einer Altersund Geschlechtsstandardisierung neutralisieren Augurzky et al. demografische Einflussfaktoren und vergleichen die Fallzahlentwicklung in verschiedenen Diagnosekategorien (Major Diagnostic Categories) der Jahre 2006 bis 2010 miteinander. Die Autoren kommen zu dem Schluss, dass demografische Faktoren rund $40 \%$ des Fallzahlwachstums erklären, wobei dieser Anteil je nach Kategorie zwischen 20 und $70 \%$ schwankt (Augurzky et al. 2012). Insgesamt schlussfolgern die Autoren, dass der Anstieg der Fallzahlen und des Casemix Index überwiegend auf einen Preisanstieg und somit auf angebotsseitige Faktoren zurückzuführen sei.

Im Rahmen des Gutachtens zur Untersuchung der Mengenentwicklung beziehen Schreyögg et al. (2014) zusätzlich zur Bevölkerungsentwicklung die Veränderungen der Mortalität und Morbidität als nachfragerelevante Indikatoren mit ein. Hierfür ziehen sie neben den Daten aus $\$ 21$ KHEntgG auch die Bevölkerungsstatistik sowie die Sterbefallstatistik der statistischen Landesämter und die ambulanten Diagnosedaten aus $₫ 295$ SGB V heran. Die Wirkungen werden auch nach verschiedenen MDCs analysiert. Es zeigte sich, dass die Nachfrageseite in den betrachteten Jahren 2007 bis 2011 einen Einfluss auf die Fallzahlentwicklung aufweist. Der Einfluss variierte deutlich zwischen den MDCs. Bei MDCs mit besonders starkem absoluten und relativen Wachstum (MDC 5 und 8) zeigte sich ein schwächerer Einfluss der Nachfrage als im Durchschnitt über alle MDCs (Schreyögg et al. 2014).

\section{- Unter den Teilkomponenten}

der Nachfrageseite spielen Morbiditäts-

veränderungen die größte Rolle

In einer aktuellen Studie erweitern Krämer und Schreyögg (2019) den methodischen Ansatz zur Analyse des Beitrags der Nachfrageseite auf die Fallzahlentwicklung. Auch hier werden die Bevölkerungsentwicklung, Veränderungen der Morbidität und Mortalität als nachfrageseitige Determinanten der Fallzahlsteigerung einbezogen (2007 bis 2011). Es zeigt sich, dass die nachfrageseitigen Determinanten über alle MDCs hinweg insgesamt ca. $20 \%$ des Fallzahlanstiegs erklären. Die Studie kann außerdem zeigen, dass innerhalb der Indikatoren auf der Nachfrageseite die Veränderung der Morbidität insgesamt den größten Effekt auf die Mengenentwicklung hat. Die Bevölkerungsentwicklung spielt hingegen eine untergeordnete Rolle. Die Studie ermöglicht auch eine Differenzierung des Effekts nach verschiedenen Altersgruppen. Die Bevölkerungsentwicklung nimmt in Altersgruppen unter 80 Jahren den zweitwichtigsten Stellenwert ein, während der Todeszeitpunkt, hier erfasst durch das letzte Jahr vor dem Tod, die zweitgrößte Rolle in Altersgruppen über 80 Jahren spielt (Krämer und Schreyögg 2019).

Insgesamt deutet die vorhandene Evidenz darauf hin, dass die Fallzahlentwicklung nicht allein durch Veränderungen der Nachfrageseite erklärt werden kann. Dennoch hat die Nachfrageseite einen gewissen Einfluss auf die Fallzahlsteigerungen und dabei insbesondere die Morbiditätsentwicklung. Der Anteil, den die Nachfrageseite an der Mengenentwicklung nimmt, ist dabei offensichtlich stark von der jeweiligen Major Diagnostic Group abhängig (Augurzky et al. 2012; Schreyögg et al. 2014; Krämer und Schreyögg 2019).

Als zweiten Erklärungsstrang für die Fallzahländerung bieten sich von der Angebotsseite gesteuerte Veränderungen in der Leistungserbringung an, die nicht durch externe Veränderungen der Nachfrageseite erklärt werden können. Dies kann sich auf verschiedene Weisen äußern. Zum einen können die Länder 
die Angebotsstruktur durch Änderungen in der Landeskrankenhausplanung nach $\$ 108$ SGB V verändern. Zum anderen kann die Krankenhausvergütung, insbesondere das DRG-System und dessen Änderungen, die Fallzahlentwicklung beeinflussen.

\section{- Effekte der Krankenhausstruktur- entwicklung und Landeskrankenhaus- planung auf die Fallzahlentwicklung sind ungeklärt}

Bislang liegen kaum Untersuchungen darüber vor, inwieweit sich Veränderungen in der Krankenhausstruktur, der Landeskrankenhausplanung und bei der Investitionskostenförderung auf die Mengenentwicklung auswirken. Die DRG-Begleitforschung listet diverse Änderungen in der Struktur der Krankenhausversorgung auf, darunter eine Abnahme der Zahl der Krankenhäuser bei gleichbleibender Bettenzahl und Verschiebungen in den Fachabteilungen (Fürstenberg et al. 2013). Es ist jedoch nicht klar, ob diese Veränderungen mit dem Fallpauschalensystem zusammenhängen beziehungsweise ob und wie sich dies in den erbrachten Krankenhausleistungen niederschlägt. Da die Zahl der Krankenhäuser auch vor Einführung des DRG-Systems abgenommen hat (Statistisches Bundesamt 2018), ist hier kein kausaler Einfluss des Fallpauschalensystems zu erwarten. Schreyögg et al. (2014) zeigen außerdem einen starken Zusammenhang zwischen der Fallzahl und der Verweildauer auf. Daher ist denkbar, dass Krankenhäuser auf eine Bettenreduktion mit einer Absenkung der Verweildauer reagieren, um die Fallzahl ausweiten zu können.

\section{- - Veränderung der Relativgewichte bewirkt Fallzahländerung, die Wirkung der Veränderungen von Basisfallwerten ist unklar}

Veränderungen des Landesbasisfallwertes haben vermutlich einen geringen Einfluss auf die Mengenentwicklung. Augurzky et al. (2012) finden in ihrem Gutachten zur Mengenentwicklung keinen Zusammenhang und führen an anderer Stelle aus, dass Basisfallwerte die heterogenen Kostenstrukturen von Krankenhäusern nicht abbilden (Augurzky und Schmitz 2013). Schreyögg et al. (2014) finden einen positiven Zusammenhang zwischen einer Basisfallwertsteigerung und einer Fallzahlerhöhung. Dieser Zusammenhang wird allerdings von den Autoren als nicht belastbar eingeschätzt, unterem anderen da die Datenjahre 2005 und 2006, die eine besonders hohe Varianz aufweisen, nicht einbezogen werden konnten.

Demgegenüber kann mit robusten Ergebnissen gezeigt werden, dass sich Veränderungen der Relativgewichte auf die Fallzahlentwicklung auswirken. Schreyögg et al. (2014) können unter Verwendung der Abrechnungsdaten nach $\$ 21$ KHEntgG nachweisen, dass ein Anstieg des Relativgewichts einer DRG in einen Fallzahlanstieg mündet. Durchschnittlich führte ein einprozentiger Anstieg des Relativgewichts einer DRG zu einer Fallzahlerhöhung um $0,2 \%$. Letztere Erkenntnis fügt sich in die internationale Literatur ein, die nachweisen konnte, dass Preissteigerungen zu vermehrter Inanspruchnahme führen (Dafny 2005). Martinussen und Hagen (2009) und Schreyögg et al. (2014) finden außerdem einen Zusammenhang zwischen der Veränderung der Kalkulationsstichprobe des InEK und Veränderungen in der Leistungserbringung von Krankenhäusern. Das heißt, dass eine Änderung der Leistungserbringung nicht nur durch sich verändernde Kostenstrukturen, sondern auch durch den $\mathrm{Zu}$ - und Abgang von Krankenhäusern, die ihre Kostendaten beim InEK einreichen, determiniert wird.

\section{- Erste Hinweise auf Upcoding}

Erste Untersuchungen zeigen Tendenzen zu Upcoding von Fällen in finanziell attraktiveren Fallpauschalen für Teile des Leistungsspektrums in Deutschland. Für die Neonatologie zeigen Jürges und Köberlein (2015), dass Krankenhäuser einen Spielraum bei der Absenkung des Geburtsgewichts nutzen, um Neugeborene in die nächsthöhere Fallpauschale einzugruppieren. Sie nutzen Daten der deutschen Geburtsstatistik der Jahre 1996 bis 2010 und der strukturierten Qualitätsberichte von 2006 
bis 2011. Den Autoren zufolge wurden 12.000 Frühgeborene in eine lukrativere DRG hochkodiert, was Zusatzausgaben von 100 Mio. $€$ entspricht. Reif et al. (2018) bestätigen dies für die Jahre 2005 bis 2011 unter Verwendung von Abrechnungsdaten der Krankenhäuser und zeigen zudem, dass höhere Erlöse keine Auswirkungen auf die Intensität der Pflege haben. Die Autoren bestätigen damit eine von Abler et al. (2011) aufgeworfene Vermutung, die ein Upcoding durch den starken Anstieg von Neugeborenen mit geringen Geburtsgewichten nach Einführung des DRG-Systems bemerkt hatten.

Augurzky et al. (2012) finden ebenso einen Verdacht auf ein Upcoding von Patientinnen und Patienten, indem sie davon ausgehen, dass Krankenhäuser die Fallzahl in der höher vergüteten DRG derselben DRG-Gruppe ausweiten. Einen überproportionalen Zuwachs in ausgewählten DRGs sehen sie als Hinweis auf Upcoding. Dies erfolgt jedoch unter Verwendung von aggregierten Daten und es bleibt unklar, ob es sich um veränderte Kodieranforderungen oder Lerneffekte handelt. Schönfelder et al. (2009) rechneten die durch Upcoding entstandenen Kosten für den Zeitraum von 2004 bis 2009 auf 1,9 bis 3,24 Mrd. $€$ hoch. Hierfür verwendeten sie in den 1980ern ermittelte Anteile des Upcodings am Gesamtfallwachstum aus den USA und übertrugen sie auf zum Teil hochgerechnete, aggregierte Kostendaten der statistischen Bundesämter. Die Begleitforschung lässt die Frage nach einem möglichen Upcoding weitestgehend unbeantwortet (Fürstenberg et al. 2011, 2013).

Diese ersten Untersuchungen zu Upcoding in Deutschland bestätigen internationale Erfahrungen. In den Vereinigten Staaten ist die Upcoding-Praxis von Krankenhäusern als Ergebnis der Anreize des DRG-Systems bereits umfassend nachgewiesen worden (Silverman und Skinner 2004; Dafny 2005). Für Portugal und Italien konnte sie ebenso bestätigt werden, wobei die Autoren die finanziellen Verluste als gering einstufen (Barros und Braun 2017; Berta et al. 2010).

\subsection{Untersuchung der Auswirkungen des Fallpauschalensystems auf die technische und Kosteneffizienz}

Die Struktur des Fallpauschalensystems setzt bewusst Anreize zur Verbesserung der Effizienz, indem es die Krankenhäuser honoriert, die dieselbe Leistung ressourcenärmer erbringen als ihre Mitstreiter. Ob sich diese Effekte in Deutschland entfaltet haben, ist unklar. Angesichts gestiegener Fallzahlen und gesunkener Verweildauern legen die aggregierten Daten diese Vermutung sehr nahe. Es ist unwahrscheinlich, dass die Zunahme personeller Ressourcen, vornehmlich des ärztlichen Personals, den Effizienzgewinn neutralisiert. Gleichzeitig ist denkbar, dass die Umstellung der Normierung $^{1}$ im Jahr 2006 die Effizienzanreize des Fallpauschalensystems gemindert hat (Schreyögg 2017b). Die einzige bisher vorhandene empirische Studie deutet zunächst auf das Gegenteil hin. Herwartz und Strumann (2014) stellten eine signifikante Verschlechterung der technischen Effizienz fest. In ihrer Analyse wählten die Autoren allerdings einen Beobachtungszeitraum von 1995 bis 2006, der bereits kurz nach der verpflichtenden Einführung des Fallpauschalensystems endet. Es ist unwahrscheinlich, dass sich die zu erwartenden Effekte des neuen Vergütungssystems in dieser Zeit schon vollständig entfalten konnten. Die Fallzahlentwicklung ist im Jahr 2005 beispielsweise negativ und verzeichnet erst im Jahr 2006 wieder eine positive Wachstumsrate. Zudem werden die Ergebnisse der Autoren durch das parallel modifizierte ambulante Operieren verzerrt.

International ist die Evidenz zur Verbesserung der Effizienz uneindeutig und vom Länderkontext abhängig. In Norwegen, Portugal

\footnotetext{
In der seit 2005/2006 geltenden Normierung wird die absolute Summe des nationalen CasemixVolumens konstant gehalten. Dies zieht so genannte Katalogeffekte - die unterschiedliche Bewertung identischer Fälle in unterschiedlichen G-DRGVersionen - mit sich.
} 
und Schweden konnten Forscher positive Auswirkungen von Fallpauschalen auf die technische Effizienz der Leistungserbringung feststellen (Gerdtham et al. 1999a, 1999b; Gonçalves und Barros 2013; Dismuke und Sena 1999; Biørn et al. 2003). In der Schweiz lassen sich leichte Zuwächse in der Kosteneffizienz ausmachen (Widmer 2015). Die Effizienzgewinne ließen sich entweder durch einen geringeren Ressourceneinsatz - beispielsweise kürzere Verweildauern und weniger Personaleinsatz -, eine Steigerung der Fallzahlen oder beides zurückführen. In anderen Industrienationen hingegen lässt sich kein Effekt finden. In Österreich und den Vereinigten Staaten ist die erhoffte Steigerung der technischen Effizienz bisher ausgeblieben (Sommersguter-Reichmann 2000; Borden 1988; Chern und Wan 2000).

\subsection{Auswirkungen des Fallpauschalensystems auf die Behandlungsqualität}

Grundsätzlich wurde das Fallpauschalensystem eingeführt, um die Wirtschaftlichkeit, Transparenz und Effizienz des Krankenhauswesens zu verbessern (Deutscher Bundestag 2001, O'Reilly et al. 2012). Diese positiven Anreize können negative Auswirkung auf die Behandlungsqualität nehmen. Dazu zählen die bevorzugte Aufnahme von bestimmten Patientengruppen (cream-skimming), das Unterlassen notwendiger, aber kostenintensiver Prozesse sowie die medizinisch verfrühte Entlassung von Patienten (bloody discharges), um einem Abschlag bei Kurzliegern zu entgehen beziehungsweise um neue Patienten aufzunehmen. Dies kann sich in höheren Mortalitätsraten während oder nach dem stationären Aufenthalt äußern sowie zu höheren Wiedereinweisungsraten führen.

Die Auswirkungen der Einführung des Fallpauschalensystems auf die Behandlungsqualität ist in Deutschland weitestgehend unerforscht. Daher können derzeit kaum belastbaren Aussagen dazu getroffen werden, ob das
Fallpauschalensystem einen positiven, negativen oder keinen Effekt auf die Qualität der Leistungserbringung genommen hat. Die wenigen Untersuchungen, die sich dieser Frage angenommen haben, basieren auf den strukturierten Qualitätsberichten. Die Begleitforschung des Fallpauschalensystems stellt einen generellen Rückgang der poststationären Mortalität zwischen 2004 und 2010 fest, jedoch ist nicht klar, ob dies durch das Fallpauschalensystem beeinflusst wurde, da keine Kontrollgruppe existiert. Zugleich verbleibt unklar, ob sich die Mortalität je nach Diagnosegruppe unterschiedlich entwickelt hat (Fürstenberg et al. 2013, 2011).

Der internationale Forschungsstand ergibt kein eindeutiges Bild (O'Reilly et al. 2012). Unklar ist zunächst, ob es zu einer bevorzugten Aufnahme lukrativer Patienten kommt. Für Norwegen konnte dieser Verdacht bei leichteren orthopädischen Diagnosen nachgewiesen werden, indem diesen Patientengruppen ein Behandlungsvorzug gegeben wurde (Martinussen und Hagen 2009). Dies wird durch Ergebnisse aus England unterstützt. Papanicolas und McGuire (2015) stellten fest, dass die Einführung des Fallpauschalensystems zu einer Ausweitung einer finanziell attraktiven Hüft-TEPProzedur gegenüber der weniger hoch vergüteten, aber von den Leitlinien empfohlenen Prozedur geführt hat. In England und Norwegen weisen Untersuchungen außerdem auf Verlagerungen der Leistungserbringung von stationären Behandlungen zu Tagesfällen (Martinussen und Hagen 2009; O'Reilly et al. 2012) sowie in den USA auf Verlegungen aus dem Krankenhaus zu kostenärmeren Leistungserbringern hin (Sager et al. 1989). Es bleibt aber unklar, ob dies zu Qualitätseinbußen führt. USbasierte Studien legen die Vermutung einer verfrühten Entlassung nahe (Rogers et al. 1990; Kosecoff et al. 1990). Kosecoff et al. (1990) zeigen beispielsweise auf, dass die Rate an instabil entlassenen Patienten in den ersten drei Jahren nach Einführung des Fallpauschalensystems von $10 \%$ auf $15 \%$ gestiegen ist.

Bezüglich der Ergebnisindikatoren ergibt sich international erneut ein gemischtes Bild. 
Grundsätzlich zeigt sich nach der Einführung des Fallpauschalensystems weder eine Qualitätsverbesserung noch eine -verschlechterung. Die Untersuchungen aus England und den USA wenige Jahre nach Einführung des Fallpauschalensystems kommen grundsätzlich zu dem Schluss, dass die Qualität konstant geblieben ist (Davis und Rhodes 1988; Rich und Freedland 1988; Farrar et al. 2009). In Frankreich konnte kein Einfluss des Fallpauschalensystems auf Wiedereinweisungen nach chirurgischen Eingriffen gefunden werden. Letztere Rate hat über die vergangenen Jahre zwar zugenommen, dies ist jedoch nicht durch das Fallpauschalensystem hervorgerufen worden (Vuagnat et al. 2018). Ein unklares Bild zeigen währenddessen die Schweiz und Japan auf. Hier wird die Einführung des Fallpauschalensystems mit geringeren Mortalitätsraten im Krankenhaus, aber gestiegenen Wiedereinweisungsraten im Krankenhaus in Verbindung gebracht (Kutz et al. 2019; Hamada et al. 2012).

\subsection{Diskussion: Die Auswirkungen der DRG-Einführung bleiben bei zahlreichen Indikatoren unklar}

Der bisherige Wissensstand bezüglich der Wirkungen des G-DRG-Systems ist in weiten Teilen uneindeutig. Als weitestgehend gesichert darf der mäßige Einfluss der Nachfrageseite auf die Mengenentwicklung gelten. Die genaue Effektstärke variiert dabei stark zwischen den Diagnosegruppen (Major Diagnostic Groups) (Schreyögg et al. 2014; Augurzky et al. 2012). Ebenso darf als gesichert gelten, dass Veränderungen der Angebotsseite einen stärkeren Einfluss auf die Fallzahlentwicklung nehmen. Hierbei kommt es sowohl zu einer durch Preisänderungen induzierten Mengenausweitung als auch zu Upcoding (Jürges und Köberlein 2015; Reif et al. 2018). Beides bestätigt die internationale Literatur (Silverman und Skinner 2004; Dafny 2005; Barros und Braun 2017).
Im Gegensatz hierzu bleibt es relativ unklar, ob die Einführung des Fallpauschalensystems zu einer Verbesserung der technischen Effizienz oder Kosteneffizienz geführt hat. Ebenso ist nicht bekannt, ob die Einführung des Fallpauschalensystems einen Einfluss auf die Behandlungsqualität genommen hat. Auch die Evidenz in der internationalen Literatur ist in den letzteren Bereichen heterogen (O'Reilly et al. 2012). International zeigen sich Hinweise auf cream-skimming und vorzeitige Entlassungen, beziehungsweise bloody discharges sowie höhere Wiedereinweisungsraten, aber geringe Effekte auf die Mortalität. Die mangelnde Evidenz, insbesondere hinsichtlich der Auswirkungen auf die Versorgungsqualität, macht eine ganzheitliche Bewertung des Fallpauschalensystems rund 15 Jahre nach seiner Einführung schwierig.

\section{- Hürden beim Datenzugang sowie fehlende Kontrollgruppe als Ursache für Mangel an Evidenz}

Die unbefriedigende Studienlage ist auf zahlreiche Gründe zurückzuführen. Erstens wurde das neue Vergütungssystem zunächst auf freiwilliger Basis und anschließend bundesweit verpflichtend eingeführt. Eine randomisierte Kontrollgruppe von Krankenhäusern, die das DRG-System zunächst nicht einführten, existiert nicht. Zweitens beginnt die diagnosebezogene Fallpauschalenstatistik erst mit Einführung des Fallpauschalensystems. Dies erschwert Vergleiche vor und nach Einführung des Fallpauschalensystems bezüglich zu betrachtender Indikatoren. Beispielsweise kann somit nur eine unzureichende Risikoadjustierung für Qualitätsvergleiche vor und nach Einführung vorgenommen werden. Drittens ist der Zugang zu den Daten nach $₫ 21$ KHEntgG über das Forschungsdatenzentrum des Statistischen Bundesamtes für komplexere Analysen kaum oder nur sehr schwer nutzbar (siehe hierzu im Detail z. B. Schreyögg 2017a). Gleichzeitig beinhaltet dieser Datensatz nur die stationäre Perspektive und z. B. keine post-stationäre Mortalität. Der Datensatz nach $₫ 303$ SGB V beim DIMDI enthält bisher keine Kranken- 
hausinstitutionskennzeichen (IK) und ist somit für solche Analysen nicht nutzbar. Dieses Problem wird mit dem Digitale-Versorgungs-Gesetz angegangen. Die künftige Nutzbarkeit dieses Datensatzes zur Adressierung der hier skizzierten Fragestellungen wird maßgeblich davon abhängen, ob das Gesetz so umgesetzt wird, dass Forscher - etwa über eine VPN-Verbindung einen Zugriff auf den Volldatensatz erhalten. Nur mit einem solchen Zugang können komplexere Modelle geschätzt werden, die in diesem Kontext erforderlich sind. Viertens sind die Daten der Kalkulationsstichprobe nicht für Forschungszwecke geöffnet. Auch die Begleitforschung konnte diesen Datensatz nicht nutzen, der für zahlreiche Fragestellungen relevant ist.

Die dargestellten Hürden für die Analyse der Wirkungen des DRG-Systems unterstreichen die Notwendigkeit, einen Zugang zu Individualdaten auf internationalem Niveau zu schaffen. Das Ziel sollte die kassenübergreifende Zusammenführung der Abrechnungsdaten aus den unterschiedlichen Sektoren des Gesundheitssystems sein. Dies ermöglicht es der Forschung, politischen Entscheidungen mit wissenschaftlicher Evidenz zur Seite zu stehen, durchgeführte Reformen zu überprüfen und eventuelle Nachadjustierungen vorzunehmen. Das Digitale-Versorgungs-Gesetz hat hierfür die gesetzlichen Voraussetzungen geschaffen.

Darüber hinaus sollte künftig bei ähnlichen Politikinterventionen eine gezielte und behutsame Pilotierung mit einer randomisierten Kontrollgruppe erfolgen. Die stufenweise Einführung der Fallpauschalenvergütung in den Schweizer Kantonen (Widmer 2015; Kutz et al. 2019) und das Experimentieren mit verschiedenen Fallpauschalensystemen in Norwegen (Kjerstad 2003) erlauben heute einen Vergleich der Wirkung von DRG-Systemen mit anderen Vergütungsmodalitäten und ermöglichen eine sauberere Trennung von kausalen Effekten des Vergütungssystems und anderen Entwicklungen.

\section{Literatur}

Abler S, Verde P, Stannigel H, Mayatepek E, Hoehn T (2011) Effect of the introduction of diagnosis related group systems on the distribution of admission weights in very low birthweight infants. Arch Dis Child 96(3):F186. https://doi.org/10.1136/adc.2010. 192500

AOK-Bundesverband, BKK Bundesverband, IKKBundesverband, See-Krankenkasse, Bundesverband der landwirtschaftlichen Krankenkassen, Bundesknappschaft, Verband der Angestellten-Krankenkassen e. V., AEV-ArbeiterErsatzkassenverband, Verband der Privaten Krankenversicherung, Deutsche Krankenhausgesellschaft (2004) Vereinbarung zum Fallpauschalensystem für Krankenhäuser für das Jahr 2005 (Fallpauschalenvereinbarung 2005 - FPV 2005). AOK-Bundesverband, BKK Bundesverband, IKK-Bundesverband, See-Krankenkasse, Bundesverband der landwirtschaftlichen Krankenkassen, Bundesknappschaft, Verband der Angestellten-Krankenkassen e. V., AEV-ArbeiterErsatzkassenverband, Verband der Privaten Krankenversicherung, Deutsche Krankenhausgesellschaft, Berlin, Bonn, Essen, Bergisch Gladbach, Hamburg, Kassel, Bochum, Siegburg, Köln

Augurzky B, Gülker R, Mennicken R, Felder S, Meyer S, Wasem J et al (2012) Mengenentwicklung und Mengensteuerung stationärer Leistungen. Endbericht. Forschungsprojekt im Auftrag des GKVSpitzenverbandes

Augurzky B, Schmitz H (2013) Wissenschaftliche Untersuchung zu den Ursachen unterschiedlicher Basisfallwerte der Länder als Grundlage der Krankenhausfinanzierung. Forschungsprojekt im Auftrag des Bundesministeriums für Gesundheit. RheinischWestfälisches Institut für Wirtschaftsforschung, Essen

Barros P, Braun G (2017) Upcoding in a National Health Service: the evidence from Portugal. Health Econ 26(5):600-618. https://doi.org/10.1002/hec.3335

Berta P, Callea G, Martini G, Vittadini G (2010) The effects of upcoding, cream skimming and readmissions on the Italian hospitals efficiency: a population-based investigation. Econ Model 27(4):812-821. https:// doi.org/10.1016/j.econmod.2009.11.001

Biørn E, Hagen TP, Iversen T, Magnussen J (2003) The effect of activity-based financing on hospital efficiency: a panel data analysis of DEA efficiency scores 1992-2000. Health Care Manag Sci 6(4):271-283. https://doi.org/10.1023/A:1026212820367

Blum K, Offermanns M (2012) Einflussfaktoren des Fallzahl- und Case Mix-Anstieges in deutschen Krankenhäusern. Gutachten. Deutsches Krankenhausinstitut (DKI), Deutsche Krankenhausgesellschaft 
(DKG). Deutsches Krankenhausinstitut e. V., Düsseldorf

Borden JP (1988) An assessment of the impact of diagnosis-related group (DRG)-based reimbursement on the technical efficiency of New Jersey hospitals using data envelopment analysis. J Account Public Policy 7(2):77-96. https://doi.org/10. 1016/0278-4254(88)90012-9

Chern J-Y, Wan TTH (2000) The impact of the prospective payment system on the technical efficiency of hospitals. J Med Syst 24(3):159-172. https://doi.org/10. 1023/A:1005542324990

Coulam RF, Gaumer GL (1992) Medicare's prospective payment system: a critical appraisal. Health Care Financ Rev 1991(Suppl):45-77 (https://www.ncbi.nlm. nih.gov/pubmed/25372306. Zugegriffen: 14. November 2019)

Dafny LS (2005) How do hospitals respond to price changes? Am Econ Rev 95(5):1525-1547. https://doi.org/ $10.1257 / 000282805775014236$

Davis CK, Rhodes DJ (1988) The impact of DRGs on the cost and quality of health care in the United States. Health Policy 9(2):117-131. https://doi.org/10.1016/ 0168-8510(88)90029-2

Deutscher Bundestag (1999) Gesetzentwurf der Fraktionen SPD und BÜNDNIS90/DIE GRÜNEN. Entwurf eines Gesetzes zur Reform der gesetzlichen Krankenversicherung ab dem Jahr 2000 (GKVGesundheitsreform 2000). Drucksache 14/1245

Deutscher Bundestag (2001) Gesetzentwurf der Fraktionen SPD und BÜNDNIS 90/DIE GRÜNEN. Entwurf eines Gesetzes zur Einführung des diagnoseorientierten Fallpauschalensystems für Krankenhäuser (Fallpauschalengesetz - FPG). Drucksache $14 / 6893$

Deutscher Bundestag (2002) Antwort der Bundesregierung auf die Kleine Anfrage der Abgeordneten Dr. Hans Georg Faust, Wolfgang Lohmann (Lüdenscheid), Dr. Wolf Bauer, weiterer Abgeordneter und der Fraktion der CDU/CSU. Drucksache 14/9810 2002

Deutscher Bundestag (2004) Gesetzesentwurf der Fraktionen SPD und BÜNDNIS 90/DIE GRÜNEN. Entwurf eines Zweiten Gesetzes zur Änderung der Vorschriften zum diagnose-orientierten Fallpauschalensystem für Krankenhäuser und zur Änderung anderer Vorschriften (Zweites Fallpauschalenänderungsgesetz - 2. FPÄndG). Drucksache 15/3672.

Deutscher Bundestag (2008) Gesetzentwurf der Bundesregierung. Entwurf eines Gesetzes zum ordnungspolitischen Rahmen der Krankenhausfinanzierung ab dem Jahr 2009 (Krankenhausfinanzierungsreformgesetz - KHRG). Drucksache 16/10807

Deutscher Bundestag (2015) Gesetzentwurf der Fraktionen CDU/CSU und SPD. Entwurf eines Gesetzes zur Reform der Strukturen der Krankenhausversorgung (Krankenhausstrukturgesetz - KHSG). Drucksache $18 / 5372$
Dismuke CE, Sena V (1999) Has DRG payment influenced the technical efficiency and productivity of diagnostic technologies in Portuguese public hospitals? An empirical analysis using parametric and non-parametric methods. Health Care Manag Sci 2(2):107-116. https://doi.org/10.1023/A: 1019027509833

Ellis RP, McGuire TG (1996) Hospital response to prospective payment: Moral hazard, selection, and practicestyle effects. J Health Econ 15(3):257-277. https:// doi.org/10.1016/0167-6296(96)00002-1

Farrar S, Yi D, Sutton M, Chalkley M, Sussex J, Scott A (2009) Has payment by results affected the way that English hospitals provide care? Difference-indifferences analysis. BMJ 339:b3047. https://doi.org/ 10.1136/bmj.b3047

Feder J, Hadley J, Zuckerman S (1987) How did medicare's prospective payment system affect hospitals? N Engl J Med 317(14):867-873. https://doi.org/10. 1056/NEJM198710013171405

Fürstenberg T, Laschat M, Zich K, Klein S, Gierling P, Nolting H-D, Schmidt T (2011) G-DRG-Begleitforschung gemäß § 17b Abs. 8 KHG. Endbericht des zweiten Forschungszyklus (2006 bis 2008). IGES Institut, Berlin (Untersuchung im Auftrag des deutschen DRGInstituts (InEK))

Fürstenberg T, Laschat M, Zich K, Klein S, Gierling P, Nolting H-D, Schmidt T (2013) G-DRG-Begleitforschung gemäß § 17b Abs. 8 KHG. Endbericht des dritten Forschungszyklus (2008 bis 2010). IGES Institut, Berlin (Untersuchtung im Auftrag des deutschen DRGInstituts (InEK).)

Gerdtham U-G, Löthgren M, Tambour M, Rehnberg C (1999a) Internal markets and health care efficiency: a multiple-output stochastic frontier analysis. Health Econ 8(2):151-164. https://doi.org/10.1002/ (SICI) 1099-1050(199903)8:2\%3C151::AID-HEC411 \%3E3.0.CO;2-Q

Gerdtham U-G, Rehnberg C, Tambour M (1999b) The impact of internal markets on health care efficiency: evidence from health care reforms in Sweden. Appl Econ 31(8):935-945. https://doi.org/10.1080/ 000368499323652

Gonçalves R, Barros PP (2013) Economies of scale and scope in the provision of diagnostic techniques and therapeutic services in Portuguese hospitals. Appl Econ 45(4):415-433. https://doi.org/10.1080/ 00036846.2011 .605758

Hamada H, Sekimoto M, Imanaka Y (2012) Effects of the per diem prospective payment system with DRGlike grouping system (DPC/PDPS) on resource usage and healthcare quality in Japan. Health Policy 107(2):194-201. https://doi.org/10.1016/j.healthpol. 2012.01.002

Herwartz H, Strumann C (2014) Hospital efficiency under prospective reimbursement schemes: an empirical assessment for the case of Germany. Eur J Health 
Econ 1(2):175-186. https://doi.org/10.1007/s10198013-0464-5

Jürges H, Köberlein J (2015) What explains DRG upcoding in neonatology? The roles of financial incentives and infant health. J Health Econ 43:13-26. https://doi. org/10.1016/j.jhealeco.2015.06.001

Kahn KL, Keeler EB, Sherwood MJ, Rogers WH, Draper D, Bentow SS et al (1990) Comparing outcomes of care before and after implementation of the DRG-based prospective payment system. JAMA 264(15):1984-1988. https://doi.org/10.1001/ jama.1990.03450150084036

KFPV Verordnung zum Fallpauschalensystem für Krankenhäuser (KFPV) vom 19. September 2002 (BGBI I $\mathrm{Nr} 68$ S 3674)

KFPV 2004 Verordnung zum Fallpauschalensystem für Krankenhäuser für das Jahr 2004 (Fallpauschalenverordnung 2004) vom 13. Oktober 2003 (BGBI I Nr 51 S 1995)

KFPV 2005 Verordnung zur Bestimmung vorläufiger Landes-Basisfallwerte im Fallpauschalensystem für Krankenhäuser für das Jahr 2005 (Fallpauschalenverordnung 2005) vom 12. Mai 2005 (BGBI I Nr 28 S 1335)

Kjerstad E (2003) Prospective funding of general hospitals in Norway-incentives for higher production? Int J Health Care Finance Econ 3(4):231-251. https:// doi.org/10.1023/A:1026084304382

Kosecoff J, Kahn KL, Rogers WH, Reinisch EJ, Sherwood MJ, Rubenstein LV et al (1990) Prospective payment system and impairment at discharge: the "quicker-and-sicker" story revisited. JAMA 264(15):1980-1983. https://doi.org/10.1001/jama. 1990.03450150080035

Krämer J, Schreyögg J (2019) Demand-side determinants of rising hospital admissions in Germany: the role of ageing. Eur J Health Econ 20(5):715-728. https://doi. org/10.1007/s10198-019-01033-6

Kumar A, Schoenstein M (2013) Managing Hospital Volumes: Germany and Experiences from OECD Countries. OECD Health Working Papers No 64. https:// doi.org/10.1787/5k3xwtg2szzr-en

Kutz A, Gut L, Ebrahimi F, Wagner U, Schuetz P, Mueller B (2019) Association of the Swiss diagnosisrelated group reimbursement system with length of stay, mortality, and readmission rates in hospitalized adult patients. JAMA 2(2):e188332-e188332. https:// doi.org/10.1001/jamanetworkopen.2018.8332

Leber W-D, Schlottmann N, von Stackelberg J-M (2001) Die Einführung von DRGs in Deutschland - Erste Vereinbarungen der gemeinsamen Selbstverwaltung. In: Arnold M, Litsch M, Schnellschmidt H (Hrsg) Krankenhaus-Report 2000. Schwerpunkt: Vergütungsreform mit DRGs. Schattauer, Stuttgart, S 75-86

Martinussen PE, Hagen TP (2009) Reimbursement systems, organisational forms and patient selection: evidence from day. Health Econ Policy Law 4(2):139158. https://doi.org/10.1017/S1744133109004812

O'Reilly J, Busse R, Häkkinen U, Or Z, Street A, Wiley M (2012) Paying for hospital care: the experience with implementing activity-based funding in five European countries. Health Econ Policy Law 7(1):73101. https://doi.org/10.1017/S1744133111000314

Papanicolas I, McGuire A (2015) Do financial incentives trump clinical guidance? Hip Replacement in England and Scotland. J Health Econ 44:25-36. https:// doi.org/10.1016/j.jhealeco.2015.08.001

Reif S, Wichert S, Wuppermann A (2018) Is it good to be too light? Birth weight thresholds in hospital reimbursement systems. J Health Econ 59:1-25. https:// doi.org/10.1016/j.jhealeco.2018.01.007

Rich MW, Freedland KE (1988) Effect of DRGs on three-month readmission rate of geriatric patients with congestive heart failure. Am J Public Health 78(6):680-682. https://doi.org/10.2105/AJPH.78.6. 680

Roeder N, Bunzemeier H, Fiori W (2008) Ein lernendes Vergütungssystem. Vom Budgetierungsinstrument zum deutschen Preissystem. In: Klauber J, Robra BP, Schellschmidt H (Hrsg) Krankenhaus-Report 2007. Schwerpunkt: Krankenhausvergütung - Ende der Konvergenzphase? Schattauer, Stuttgart, S 23-47

Rogers WH, Draper D, Kahn KL, Keeler EB, Rubenstein LV, Kosecoff J, Brook RH (1990) Quality of care before and after implementation of the DRG-based prospective payment system: a summary of effects. JAMA 264(15):1989-1994. https://doi.org/10.1001/ jama.1990.03450150089037

Russell LB, Manning CL (1989) The effect of prospective payment on medicare expenditures. $\mathrm{N}$ Engl J Med 320(7):439-444. https://doi.org/10.1056/ NEJM198902163200706

Sachverständigenrat zur Begutachtung der Entwicklung des Gesundheitswesens (2018) Bedarfsgerechte Steuerung des Gesundheitswesens. Gutachten 2018, 1. Aufl. Medizinisch Wissenschaftliche Verlagsgesellschaft, Berlin

Sager MA, Easterling DV, Kindig DA, Anderson OW (1989) Changes in the location of death after passage of medicare's prospective payment system. N Engl J Med 320(7):433-439. https://doi.org/10. 1056/NEJM198902163200705

Schönfelder T, Balázs S, Klewer J (2009) Kosten aufgrund von DRG-Upcoding durch die Einführung der Diagnosis Related Groups in Deutschland. Heilberufe 61(3):77-81. https://doi.org/10.1007/s00058-0091516-1

Schreyögg J (2017a) Big Data: Datenbestände für Wissenschaft und Patienteninformation effektiver nutzen. Hg. v. Bertelsmann Stiftung (Der digiatle Patient). https://blog.der-digitale-patient.de/bigdata-datenbestaende-effektiver-nutzen/. Zugegriffen: 14. Nov. 2019 
Schreyögg J (2017b) Vorschläge für eine anreizbasierte Reform der Krankenhausvergütung. In: Klauber J, Geraedts M, Friedrich J, Wasem J (Hrsg) Krankenhaus-Report 2017. Schwerpunkt: Zukunft gestalten. Schattauer, Stuttgart, S 13-26

Schreyögg J (2019) Changes in hospital financing and organization and their impact on hospital performance: oxford university press pages. https://oxfordre.com/economics/view/10.1093/ acrefore/9780190625979.001.0001/acrefore9780190625979-e-380. Zugegriffen: 14. Nov. 2019

Schreyögg J, Bäuml M, Krämer J, Dette T, Busse R, Geissler $A$ (2014) Forschungsauftrag zur Mengenentwicklung nach § 17b Abs. 9 KHG. Endbericht. Hamburg Center for Health Economics (hche), Hamburg

Shleifer A (1985) A theory of yardstick competition. Rand J Econ 16(3):319-327. https://doi.org/10.2307/ 2555560

Silverman E, Skinner J (2004) Medicare upcoding and hospital ownership. J Health Econ 23(2):369-389. https://doi.org/10.1016/j.jhealeco.2003.09.007
Sommersguter-Reichmann M (2000) The impact of the Austrian hospital financing reform on hospital productivity: empirical evidence on efficiency and technology changes using a non-parametric input-based Malmquist approach. Health Care Manag Sci 3(4):309-321. https://doi.org/10.1023/A: 1019022230731

Statistisches Bundesamt (2018) Gesundheit. Grunddaten der Krankenhäuser. DeStatis, Wiesbaden

Theurl E, Winner H (2007) The impact of hospital financing on the length of stay: evidence from Austria. Health Policy 82(3):375-389. https://doi.org/10. 1016/j.healthpol.2006.11.001

Vuagnat A, Yilmaz E, Roussot A, Rodwin V, Gadreau M, Bernard A et al (2018) Did case-based payment influence surgical readmission rates in France? A retrospective study. BMJ Open 8(2):e18164. https://doi. org/10.1136/bmjopen-2017-018164

Widmer PK (2015) Does prospective payment increase hospital (in)efficiency? Evidence from the Swiss hospital sector. Eur J Health Econ 16(4):407-419. https:// doi.org/10.1007/s10198-014-0581-9

Open Access Dieses Kapitel wird unter der Creative Commons Namensnennung 4.0 International Lizenz (http:// creativecommons.org/licenses/by/4.0/deed.de) veröffentlicht, welche die Nutzung, Vervielfältigung, Bearbeitung, Verbreitung und Wiedergabe in jeglichem Medium und Format erlaubt, sofern Sie den/die ursprünglichen Autor(en) und die Quelle ordnungsgemäß nennen, einen Link zur Creative Commons Lizenz beifügen und angeben, ob Änderungen vorgenommen wurden.

Die in diesem Kapitel enthaltenen Bilder und sonstiges Drittmaterial unterliegen ebenfalls der genannten Creative Commons Lizenz, sofern sich aus der Abbildungslegende nichts anderes ergibt. Sofern das betreffende Material nicht unter der genannten Creative Commons Lizenz steht und die betreffende Handlung nicht nach gesetzlichen Vorschriften erlaubt ist, ist für die oben aufgeführten Weiterverwendungen des Materials die Einwilligung des jeweiligen Rechteinhabers einzuholen. 